\title{
ANÁLISE DA UTILIZAÇÃO DE INDICADORES DE DESEMPENHO A LUZ DO BALANCED SCORECARD: UM ESTUDO EM EMPRESAS DE CONSTRUÇÃO CIVIL NO MUNICÍPIO DE JOÃO PESSOA/PB
}

ANALYSIS OF THE USE OF PERFORMANCE INDICATORS IN LIGHT OF THE BALANCED SCORECARD: A STUDY IN CONSTRUCTION COMPANIES IN THE MUNICIPALITY OF JOÃO PESSOA/PB

RISOLENE ALVES DE MACENA DE ARAÚJO Universidade Federal da Paraíba LÍVIA MARIA DA SILVA SANTOS

Centro Universitário de João Pessoa

Recebido em 03/06/18

Avaliado pelo sistema double blind review e solicitado revisões em 29/06/18

Aceito para publicação pelo Editor Chefe Dr. Leonardo José Seixas Pinto em 31/07/18 e publicado em 28/12/18

\section{RESUMO}

O presente estudo objetivou analisar os indicadores de desempenho utilizados pelos gestores das empresas de construção civil do município de João Pessoa- PB, sob a luz do Balanced Scorecard. Para atender tal propósito, aplicou-se um questionário com os gestores dessas empresas. Para análise dos dados, fez-se uso da estatística descritiva, através da análise das distribuições de frequências dos indicadores. A amostra da pesquisa foi composta por 11 empresas do segmento, cadastradas no SINDUSCON-JP. Os resultados demonstraram que os indicadores das perspectivas processos internos, financeira e clientes são "utilizados frequentemente" pelos gestores, e os da perspectiva de aprendizagem e crescimento, na maioria das vezes, não são utilizados pelos gestores. Palavras-chave: Balanced Scorecard; Indicadores de Desempenho; Construção Civil.

\section{ABSTRACT}

The present study aimed to analyze the performance indicators used by managers of construction companies in the city of João Pessoa - PB, under the light of the Balanced Scorecard. To fulfill this purpose, a questionnaire was applied with the managers of these companies. For the analysis of the data, descriptive statistics were used, through the analysis of the frequency distributions of the indicators. The research sample was composed of 11 companies of the segment, registered in SINDUSCON-JP. The results showed that indicators of the internal, financial and customer process perspectives are "frequently used" by managers, and those from the perspective of learning and growth, most often, are not used by managers.

Keywords: Balanced Scorecard; Performance indicators; Construction. 


\section{INTRODUÇÃO}

A medição de desempenho sob a ótica do Balanced scorecard (BSC) é um assunto que vem sendo discutido por diversos autores (KAPLAN;NORTON, 1992; NEELY et al., 1996; SINK; TUTTLE, 1993; PARANJAPE; ROSSITER; PANTANO, 2006; CALLADO; JACK, 2015), em variados cenários organizacionais. O BSC, ferramenta criada com o objetivo de melhorar a avaliação de desempenho organizacional, tornou-se um grande instrumento gerencial e estratégico, tendo como premissa que a utilização de indicadores contábeis e financeiros, apenas, é inadequada, na era da informação, para mensurar o desempenho de uma empresa (KAPLAN; NORTON, 2001b).

Segundo Kardec, Flores e Seixas (2002 apud HOLANDA, 2007), os indicadores são como guias que permitem medir, não somente a eficácia das ações tomadas, como também avaliar o desempenho através da comparação das ações programadas com as realizadas; pois, como afirmam Neely et al. (1997) e Sink e Tuttle (1993), os indicadores de desempenho devem ser considerados parte integrante do processo de planejamento e controle, fornecendo meios de capturar dados que podem ser utilizados como informação para tomada de decisão nas empresas.

O BSC se diferencia de outros sistemas de gestão estratégica, pelo fato de levar à criação de uma rede de indicadores de desempenho que atingem todos os níveis organizacionais, que, por sua vez, são eficientes para comunicar e promover o comprometimento geral dos colaboradores com a estratégia da empresa (PRIETO et al., 2006). O BSC reflete "o equilíbrio entre os objetivos de curto e longo prazo, entre medidas financeiras e não financeiras, entre indicadores de tendências e ocorrências e entre as perspectivas internas e externas de desempenho" (KAPLAN; NORTON, 1997), mensuradas sob quatro perspectivas: financeira, clientes, processos internos e de aprendizado e crescimento.

As definições dos indicadores de desempenho e metas podem ser vistas como formulações concretas de escolhas estratégicas da empresa (WOULTERS; SPORTEL, 2005), e os resultados reais alcançados, para as várias medidas, sinalizam o quão bem a empresa consegue atingir essas escolhas (KAPLAN; NORTON, 2000). Dessa forma, Woulters e Sportel (2005) enfatizam que os relatórios de medição de desempenho podem incluir um grande número de medidas diferentes para cada unidade de responsabilidade, tais como: desempenho financeiro, relacionamento com clientes, processos internos do negócio, aprendizado e objetivos de crescimento da empresa e os funcionários.

Observa-se, no entanto, deficiências teóricas do BSC, ao sugerir relação de causa e efeito, empregando um modelo de controle de cima para baixo, para implementação que parece ignorar as realidades organizacionais (NORREKLIT, 2000). Van Aken et al. (2005) acrescentam que o BSC é "grande na teoria", porém, identifica-se uma série de problemas na sua implementação e uso: "o sistema é demasiado complexo, com muitas medidas; o sistema é muitas vezes altamente subjetivo na sua aplicação efetiva; e métricas precisas, muitas vezes, perdem elementos importantes de desempenho, que são mais difíceis de quantificar, mas que podem ser cruciais para o sucesso organizacional a longo prazo".

Não obstante, adequar os critérios para escolher quais indicadores são mais adequados, para avaliar o desempenho das organizações, é uma tarefa bastante complexa, uma vez que esses critérios variam ao longo do tempo, de um setor para outro, e, até mesmo, de uma empresa para outra, sendo muito difícil sua generalização (PINTO, 2002; NAVARRO, 2005). Dessa forma, a tentativa de definilos deve considerar as especificidades de cada organização, pois não existe uma fórmula única a ser utilizada. É preciso que os indicadores sejam selecionados de acordo com os objetivos estratégicos e fatores críticos da empresa, de modo a permitir que os gestores obtenham maior capacidade de resposta e flexibilidade para a resolução de problemas (NEELY, 1999), devendo ser estruturados, no que se refere à forma de coleta, processamento, análise da mão-de-obra e utilização dos resultados (OHASHI et al., 2004).

Ao tratar das empresas de Construção Civil, percebe-se que estas exercem forte alavancagem nos setores que lhe servem de fornecedores de insumo, tais como a indústria de matéria-prima, serviços e equipamentos (KURESKI et al.,2008). Sobremaneira, a complexidade do setor advém dos vários impactos sobre os aspectos econômicos, sociais, tecnológicos e governamentais que se interrelacionam na dinâmica do segmento (FIALHO et al, 2014), conforme percebido nos dados 
apresentados pelo Instituto Brasileiro de Geografia e Estatística - IBGE (2013), o qual evidencia a indústria de construção civil como responsável por 4,8\% do PIB brasileiro no ano de 2012. Ainda, de acordo com o IBGE (2013), esse segmento da economia também possui grande importância na geração de empregos, tendo em vista que aproximadamente $7,7 \%$ das pessoas ocupadas no Brasil exerciam atividades no setor da construção civil em 2012, especialmente, profissionais menos qualificados que não teriam oportunidade de acesso a outros mercados de trabalho (SANTOS, 2015).

Contudo, verifica-se que o uso dos indicadores ainda não constitui uma prática sistemática para muitas das empresas desse segmento, apesar de ter sido identificada a adesão a alguns programas formais de melhoria de desempenho e certificação de seus sistemas de gestão da qualidade, como por exemplo, a norma NBR ISO 9000 e PBQP-H (Programa Brasileiro de Qualidade e Produtividade do Habitat) (COSTA et al, 2005). Lantelme (1994) aponta, como justificativa para este quadro, a falta de conhecimento e experiência dos diretores, gerentes e demais funcionários da construção civil, quanto à coleta, processamento e avaliação dos indicadores de desempenho.

Adicionalmente, Costa (2003) relata que as dificuldades em conceber e em implementar sistemas de indicadores de desempenho, em empresas de construção civil, também foi verificada em outros países. Na Austrália, por exemplo, foi realizado um estudo para avaliar e determinar o estágio atual dos sistemas de medição da qualidade, na construção, e as barreiras relevantes, quanto à implementação desses sistemas, e constataram que 75\% das empresas entrevistadas não tinham sistemas de medição de desempenho (BHUTA; KARKHANIS, 2002, apud COSTA, 2003); e quando existem indicadores, verifica-se que as empresas acabam por optar pela coleta de indicadores mais simples, com dados de mais fácil acesso ou que forneçam resultados em curto prazo, sem avaliar quais informações são mais relevantes para a empresa (LANTELME, 1994), ocasionando grandes problemas. Assim, surge a seguinte questão: Quais indicadores de desempenho, sob a luz do Balanced Scorecard, são utilizados pelos gestores das empresas de construção civil do município de João Pessoa/PB?

O estudo tem como objetivo analisar os indicadores de desempenho utilizados pelos gestores das empresas de construção civil do município de João Pessoa- PB, sob a luz do Balanced Scorecard. Sob esse prisma, a presente pesquisa justifica-se, primeiramente, pelo tema estar diretamente ligado ao fato do segmento possuir grande representatividade econômica, social e ambiental, os quais precisam ser acompanhados e controlados por indicadores de desempenho.

Conforme Santos (2015), a quantidade de empresas que atuam no setor cresceu de $96.867 \mathrm{em}$ 2000 para 208.537 em 2012, com reflexos na geração de emprego, renda, tributos e no fomento de setores econômicos associados, como: cimento e concreto, extração mineral, máquinas e equipamentos, produtos siderúrgicos, materiais básicos, serviços especializados para construção, entre outros (Instituto Brasileiro de Geografia e Estatística - IBGE, 2010; Câmara Brasileira da Indústria da Construção - CBIC, 2014). Dessa forma, a pesquisa justifica-se, também, pelo fato do desenvolvimento na construção civil nas últimas décadas ter aumentado a competitividade, fazendo com que empresas repensem nas estratégicas que possam reduzir os custos ocasionados por desvios em relação à meta estabelecida, e melhorarem a qualidade, vislumbrando não só os aspectos financeiros, mas também os valores intangíveis, ora contemplados pelo BSC.

Espera-se, portanto, que esta pesquisa apresente informações que venham contribuir para o crescimento do setor de construção civil, no que se refere à utilização de indicadores de desempenho pelos gestores das empresas pertencentes ao setor, no contexto organizacional, bem como identificar a frequência do uso dos indicadores pelas empresas da amostra selecionada.

\section{REVISÃO DE LITERATURA}

\subsection{Balanced scorecard (BSC) e os Indicadores de Desempenho}

Informações de medição de desempenho são utilizadas para efetuar a mudança positiva na cultura organizacional, sistemas e processos, bem como ajudar a definir metas, alocação e priorização de recursos na consecução dos objetivos da empresa. Neste sentido, Norreklit (2008) aponta que um sistema de avaliação de desempenho permite a empresa planejar, controlar e mensurar seu desempenho, de acordo com uma estratégia pré-definida. Em suma, existe uma grande influência na

ISSN 2595-7287 | RCGC | UFF | Niterói/RJ | V.1 | n.2 | jul-dez. 2018 | p. 36-51 
medição de desempenho sobre as decisões das empresas, sejam de caráter estratégico ou operacional (FOLAN; BROWNE, 2005), a alcançarem os resultados desejados e criar valor para o acionista (MARTINS et al. 2013).

Por anos, os gestores avaliavam os resultados da empresa apenas por indicadores financeiros, sob uma perspectiva de curto prazo. Com o tempo, percebeu-se a necessidade de mensurar outros fatores, como a satisfação do cliente, desempenho dos processos internos e o desenvolvimento do capital humano da empresa, bem como direcionar seus recursos na melhoria contínua, possibilitando alcançar os objetivos estratégicos organizacionais num espaço maior de tempo, ou seja, em longo prazo, pois, segundo Kaplan e Norton (1992), os executivos entendem que as medidas de contabilidade financeira tradicionais, como o retorno sobre o investimento e o lucro por ação, podem dar sinais enganosos para a melhoria contínua e atividades de inovação demandadas no ambiente competitivo de hoje.

Contrastando a esta situação de inadequação dos sistemas de medição de desempenho, frente às novas tecnologias, estruturas organizacionais e concepções de administração, diversas propostas foram realizadas para solucionar esse obstáculo (MARTINS; COSTA, 1998). Dentre as proposta, destaca-se o Balanced scorecard, cujo modelo, desenvolvido por Kaplan e Norton (1992), resultou da necessidade de captar toda a complexidade da performance da organização, direcionando-a para o seu sucesso no futuro, ao traduzir sua missão e estratégia por um conjunto abrangente de medidas de desempenho, que serve de base para um sistema de medição e gestão estratégica, as quais são separadas em quatro dimensões: financeira, cliente, processos internos e aprendizado e crescimento.

Para Kaplan e Norton (2004, p.32), "a perspectiva financeira descreve os resultados tangíveis da estratégia em termos financeiros tradicionais", como índices de rentabilidade, crescimento de receitas e custos por produtos, giro de caixa, entre outros. Corroborando o exposto, Filgueiras, Barros e Gomes (2010) afirmam que os objetivos e medidas financeiras desempenham um papel duplo: i) definir o desempenho financeiro esperado da estratégia e servir de meta principal para a definição dos objetivos; e ii) se tornar medidas das outras perspectivas do BSC. Já a perspectiva do cliente define a proposição de valor para os clientes-alvo (KAPLAN; NORTON, 2004). Dessa forma, os gerentes das unidades de negócios articulam as estratégias de clientes e mercados, que proporcionarão maiores lucros financeiros futuros, tais como a captação e a lucratividade obtida por meio da satisfação, fidelidade e retenção de clientes (QUEIROZ et al., 2015).

A perspectiva dos processos internos, que se refere à maneira como a empresa estrutura suas atividades para entregar produtos e serviços aos seus clientes, é representada pelas seguintes medidas: (a) tempo de ciclo operacional; (b) qualidade; (c) habilidades dos funcionários; e (d) produtividade (KAPLAN; NORTON, 1992; FILGUEIRAS; BARROS; GOMES, 2010). Complementando o exposto, Kaplan e Norton (1997, p. 25) evidenciam que essa perspectiva identifica os processos internos críticos, nos quais a empresa deve alcançar a excelência, e, por conseguinte, obter excelentes retornos financeiros. A perspectiva de aprendizado e crescimento enfatiza a importância de alinhar os ativos intangíveis com a estratégia da organização (KAPLAN; NORTON, 2004). Kaplan e Norton (2000) sugerem que o aprendizado e crescimento são fundamentais para as organizações se manterem a longo prazo, pois são a base para a melhoria da qualidade, tendo em vista a competitividade. Destacam-se algumas medidas que, geralmente, são realizadas para alcançar esse objetivo: (a) lançamento de novos produtos; (b) criação de mais valor para os clientes; (c) melhoria da eficiência operacional continuamente; (d) investimento no desenvolvimento de seu pessoal; e (e) melhoria dos sistemas de informação (KAPLAN; NORTON, 1992).

Sainaghi, Phillips e Corti (2013) argumentam que a interação dessas dimensões, ao incorporarem medidas financeiras e não financeiras, devem fazer parte do sistema de informação para os funcionários em todos os níveis da organização, haja vista que um importante pressuposto, definido por Kaplan e Norton (1996), diz respeito à falta de definição de uma estratégia comum e à sua transição para objetivos claros. O conhecimento das metas da organização, por si só, não é suficiente para mudar ou influenciar o comportamento das pessoas; é necessário incentivar o entrosamento dos objetivos com as medidas, associando o nível individual às metas de longo prazo da empresa (KAPLAN; NORTON, 1996; FILGUEIRAS; BARROS; GOMES, 2010).

ISSN 2595-7287 | RCGC | UFF | Niterói/RJ | V.1 | n.2 | jul-dez. 2018 | p. 36-51 
Existem, entretanto, algumas críticas quanto à eficiência do BSC: i) o BSC deixa de lado alguns pontos fundamentais no monitoramento das relações contratuais entre agentes e principais (ATKINSON et al., 1997); ii) há predominância da ênfase no planejamento, em vez de controle (MOORAJ; OYON; HOSTETTLER, 1999); iii) problemática da defasagem de tempo entre causa e efeito das medidas de desempenho contempladas no BSC (NORREKLIT, 2000); e iv) inflexibilidade do BSC como desvantagem competitiva sustentável para as empresas que enfrentam muitos estratégias de incertezas (VEEN-DIRKS; WIJN, 2002). Segundo Paranjape, Rossiter e Pantano (2006), a solução de parte desses problemas, requer a participação dos gestores na verificação do ambiente interno e externo da empresa, ou seja, eles precisam conhecer o negócio no qual trabalham.

Diante desse cenário, houve também adequações ao modelo proposto por Kaplan e Norton (1992), uma delas foi o uso do Benchmarking. Essa proposta, de acordo com Punniyammoorthy e Murali (2008), enfatiza que o rápido desenvolvimento tecnológico e as melhorias na comunicação forçam os gerentes a lidarem com uma grande quantidade de dados para tomada de decisão, produzindo resultados comparáveis aos padrões de mercado. Porém, para o Benchmarking ocorrer, é necessário que os gestores garantam que a informação é devidamente comunicada aos funcionários, bem como realizar o acompanhamento periódico dos progressos, no que diz respeito à realização dos objetivos estratégicos. Dessa forma, os gestores terão como fazer uma avaliação significativa de suas estratégias e rever a eficiência da empresa sob todas as dimensões de suas operações.

\subsection{Estudos Correlatos}

Diversos estudos sobre BSC foram realizados no ambiente corporativo, destacando-se o de Chia, Goh e Hum (2009); Diniz, Godoy e Stefano (2012); Rocha e Casartelli (2014); Pech et al.(2015).

Com o objetivo de examinar empiricamente como os executivos de uma cadeia de suprimentos de serviços logísticos percebem a medição de desempenho por meio das perspectivas do BSC, Chia, Goh e Hum (2009) analisaram 113 entidades de serviços logísticos. Os resultados sugerem que os respondentes são focados, principalmente, na utilização de indicadores de desempenho financeiros, apesar de uma importância secundária também ser colocada em medidas de entrega e satisfação do cliente dentro do prazo. Além disso, os resultados mostraram uma aparente falta no foco sobre os condutores de desempenho estratégico futuro, como implícito nos resultados da medição de processos internos de negócio e indicadores de aprendizado e crescimento. Essas duas perspectivas contêm medidas que criam valor futuro, e abordam o desenvolvimento do núcleo competências, mas eles não foram bem medidos.

Enquanto que no trabalho de Diniz, Godoy e Stefano (2012), ao avaliarem a satisfação dos clientes internos e externos de uma cooperativa de laticínios em relação à qualidade de seus produtos e serviços, sob a perspectiva dos indicadores do BSC, concluíram que o mapa estratégico do balanced scorecard, utilizado pela empresa, estava alinhado com seus indicadores para o conhecimento amplo e detalhado dos objetivos, traduzidos, na perspectiva dos clientes, como coerentes com a satisfação identificada na pesquisa. Evidências de sucesso da aplicação do BSC também foram identificadas por Pech et al. (2015). Esses autores realizaram um estudo com o objetivo de analisar a utilização do BSC em uma Cooperativa de Transporte do Estado de Santa Catarina, e constataram que a empresa está trabalhando adequadamente, conforme prevê o modelo do BSC, proposto por Kaplan e Norton (2000).

Na tentativa de compreender as dificuldades de utilização do BSC, Rocha e Casartelli (2014) se propuseram a avaliar os fatores críticos para sua implantação em uma instituição de ensino superior (IES). Para tanto, os autores realizaram entrevistas com oito gestores que tiveram atuação importante no processo de implantação do BSC. Os resultados obtidos realçam o reconhecimento da importância do planejamento estratégico para as IES; a existência de um foco estratégico comum; a definição de prioridades institucionais abrangentes; a criação de uma equipe de implantação capaz de disseminar a metodologia do BSC; possuir uma ferramenta da tecnologia da informação para apoiar o processo; e contar com a participação ativa da alta administração. Além disso, apontam como desafios a maior 
apropriação da ferramenta pelos usuários, a integração do sistema de BSC aos demais utilizados pela Universidade e o aprofundamento da cultura de gestão estratégica de longo prazo.

Pelo exposto, é possível apontar que a utilização do BSC já vem sendo amplamente discutida na academia, assim a presente pesquisa não projeta-se para dar visibilidade as discussão das ferramentas gerenciais nas referidas instituições, mas sim complementar a literatura existente e servir como fonte de consulta a outros pesquisadores e gestores que estejam propensos a se aprofundar sobre a temática do BSC.

\section{METODOLOGIA}

A classificação da presente pesquisa, quanto ao seu objetivo, é descritiva, uma vez que foram analisadas as características das empresas das empresas de construção civil do município de João Pessoa- PB no tocante aos indicadores de desempenho utilizados pelos seus gestores.

O procedimento utilizado, para a coleta dos dados, foi a aplicação de um questionário. Este procedimento, segundo Chizzotti (1991, p.55), consiste em um "conjunto de questões pré-elaboradas, sistemática e sequencialmente dispostas em itens que constituem o tema da pesquisa, com o objetivo de suscitar dos informantes respostas por escrito ou verbalmente sobre o assunto que os informantes saibam opinar ou informar". Quanto à abordagem, a pesquisa é quantitativa, pois utilizou-se técnicas estatísticas no tratamento dos dados, tomando por base os trabalhos realizados por Holanda (2007), Chia, Goh e Hum (2009), onde foram tratadas questões ligadas à indicadores de desempenho, no setor da Construção Civil, e à frequência dos indicadores sob as quatros perspectivas do Balanced Scorecard, respectivamente.

O questionário utilizado foi adaptado da pesquisa de Holanda (2007), sendo estruturado da seguinte forma: a) Parte I: contém questões relacionadas ao perfil dos entrevistados (5 itens); b) Parte II: inclui quesitos de caracterização das empresas (6 questões); c) Parte III: contém questões sobre os aspectos relacionados à medição de desempenho ( 9 perguntas dicotômicas); d) Parte IV: visa identificar a frequência da utilização dos indicadores de desempenho (1 questão, composta por 12 itens de perspectivas de processos internos de negócios, 9 itens de perspectivas financeira, 4 itens de perspectivas de aprendizagem e crescimento e 4 itens de perspectivas de clientes, todas em escala tipo likert)

A população da pesquisa foi composta por 155 empresas do segmento da construção civil, que possuem cadastro no Sindicato da Construção Civil de João Pessoa (SINDUSCON-JP), e cujos nomes são disponibilizados no site do sindicato. Os convites para participar da pesquisa foram enviados para o e-mail dos participantes, por intermédio do SINDUSCON-JP, utilizando o Google Docs, como plataforma. Do total, apenas 11 empresas responderam o questionário aplicado, sendo este, portanto, o número de empresas pertencentes à amostra. O motivo da utilização do sindicato, como intermediador, deu-se em função da não disponibilidade, em seu portal, dos contatos eletrônicos dessas empresas, e pelo universo da pesquisa ser composto por um número significativamente alto, o que inviabilizava a localização individual das empresas, em sites de buscas.

Para atingir os objetivos propostos nesta pesquisa, foram utilizados dois métodos distintos de análise dos dados. Num primeiro momento, foram analisados os questionários para a tabulação dos mesmos em uma planilha eletrônica, para que, em seguida, se efetuasse a distribuição das frequências das variáveis contidas no questionário. Após isso, foi calculada a frequência percentual para cada resposta obtida por meio da aplicação do questionário. Dessa maneira, fez-se uso da estatística descritiva, com o intuito de expor as características dos respondentes e das empresas que compõem a amostra, bem como a análise das distribuições de frequências dos indicadores.

\section{ANÁLISE DOS RESULTADOS}

Na Tabela 1, inicialmente, é apresentado o perfil dos entrevistados, com a distribuição da frequência numérica, e em percentuais, de cada variável verificada, tais como função desempenhada na empresa, nível de escolaridade, curso e tempo de experiência na atividade. 
Tabela 1: Perfil dos Entrevistados

\begin{tabular}{lcc}
\hline Cargo desempenhado na empresa & Frequência & $\mathbf{\%}$ \\
\hline Proprietário e Gerente (Presidente) & 4 & $36 \%$ \\
Gerente Administrativo-financeiro & 3 & $27 \%$ \\
Contador & 1 & $9 \%$ \\
Outras & 3 & $27 \%$ \\
\hline Total & 11 & $100 \%$ \\
\hline Nível de escolaridade & Frequência & $\mathbf{\%}$ \\
\hline Nível Superior Completo & 11 & $100 \%$ \\
\hline Total & 11 & $100 \%$ \\
\hline Curso do Respondente & Frequência & $\mathbf{\%}$ \\
\hline Ciências Contábeis & 5 & $45 \%$ \\
Direito & 2 & $18 \%$ \\
Engenharia Civil & 3 & $27 \%$ \\
Recursos Humanos & 1 & $9 \%$ \\
\hline Total & 11 & $100 \%$ \\
\hline Tempo de experiência na atividade & Frequência & $\mathbf{\%}$ \\
\hline 1 a 5 anos & 6 & $55 \%$ \\
Acima de 5 anos até 10 anos & 4 & $36 \%$ \\
Acima de 10 anos & 1 & $9 \%$ \\
\hline Total & 11 & $100 \%$ \\
\hline Fonte: Dados & & \\
\hline
\end{tabular}

Fonte: Dados da Pesquisa, 2016

Observou-se que a maioria dos respondentes da pesquisa ocupa o cargo de Proprietário e Gerente (36\%), ou o cargo de Gerente Administrativo-Financeiro (27\%), enquanto apenas 9\% ocupam o cargo de Contador e $27 \%$ em outros cargos, todos apresentando o ensino superior completo, como grau de escolaridade. Desse total, $45 \%$ representados pelo curso de Ciências Contábeis e $27 \%$ de Engenharia Civil. Quanto ao tempo de experiência dos respondentes, na atividade que desenvolvem atualmente, $55 \%$ encontram-se entre 1 e 5 anos, $36 \%$ entre 5 e 10 anos, e apenas $9 \%$ acima de 10 anos.

Posteriormente, é apresentada na tabela 2 a distribuição das variáveis que caracterizam as empresas da amostra, demonstrando que o tempo de atuação da maioria das empresas encontra-se no intervalo de 1 a 5 anos e 5 até 10 anos, representando $36 \%$, cada, seguido dos $18 \%$ das empresas que têm acima de 20 anos de atuação e, apenas, 9\% entre 10 a 20 anos. Houve uma predominância (91\%) das empresas, cuja origem do seu capital é nacional privado e não fazem parte de grupo de empresas, e os outros $9 \%$ também é nacional privado, porém faz parte de grupo de empresas. Das empresas pesquisadas, a maioria (64\%) é administrada por familiares, ou seja, por pessoas que possuem algum grau de parentesco com o proprietário da empresa. 
Tabela 2: Perfil das empresas

\begin{tabular}{lcc}
\hline Tempo de atuação da empresa no mercado & Frequência & $\%$ \\
\hline 1 a 5 anos & 4 & $36 \%$ \\
Acima de 5 anos até 10 anos & 4 & $36 \%$ \\
Acima de 10 anos até 20 anos & 1 & $9 \%$ \\
Acima de 20 anos & 2 & $18 \%$ \\
\hline Total & Frequência & $100 \%$ \\
\hline Números de funcionários na empresa & 3 & $\%$ \\
\hline Até 19 funcionários- Microempresa & 3 & $27 \%$ \\
De 20 a 99 funcionários- Pequena empresa & 5 & $27 \%$ \\
De 100 até 499 funcionários- Grande Empresa & 11 & $45 \%$ \\
\hline Total & Frequência & $100 \%$ \\
\hline Origem do capital da empresa & 10 & $\%$ \\
\hline Nacional Privado, empresa única. & 1 & $91 \%$ \\
Nacional Privado, participante de grupo de empresas & 11 & $9 \%$ \\
\hline Total & Frequência & $100 \%$ \\
\hline Administração da empresa & 4 & $\%$ \\
\hline Profissionais contratados no mercado & 7 & $36 \%$ \\
Familiar & 11 & $64 \%$ \\
\hline Total & $100 \%$ \\
\hline
\end{tabular}

Fonte: Dados da Pesquisa, 2016

O número de funcionários foi considerado na pesquisa para caracterizar o porte da empresa, conforme critério do Instituto Brasileiro de Geografia e Estatística (IBGE) (HOLANDA, 2007). Percebe-se uma concentração maior de empresas ditas médio porte, onde o intervalo considerado foi de 100 a 499 funcionários, e estas representaram 45\% do total. As microempresas (até 19 funcionários) e as empresas de pequeno porte (entre 19 e 99 funcionários) seguem com 27\%, cada.

$\mathrm{Na}$ terceira parte, tem-se os aspectos relacionados com a medição de desempenho das empresas (tabela 3). Os pontos investigados tiveram por objetivo verificar se as empresas utilizam ou não algum tipo de indicador de desempenho nas rotinas de tomada de decisão, bem como compreender o processo de planejamento e controle estratégico.

Tabela 3: Aspectos ligados a medição de desempenho

\begin{tabular}{|c|c|c|c|c|}
\hline Alternativas & & Sim & Não & Total \\
\hline \multirow{2}{*}{ Elabora Orçamento } & Quant. & 11 & 0 & \multirow{2}{*}{11} \\
\hline & $\%$ & $100 \%$ & $0 \%$ & \\
\hline \multirow{2}{*}{ Faz comparações entre o planejado e executado } & Quant. & 11 & 0 & \multirow{2}{*}{11} \\
\hline & $\%$ & $100 \%$ & $0 \%$ & \\
\hline \multirow{2}{*}{ Possui missão definida } & Quant. & 10 & 1 & \multirow{2}{*}{11} \\
\hline & $\%$ & $91 \%$ & $9 \%$ & \\
\hline \multirow{2}{*}{$\begin{array}{l}\text { Apresenta claramente sua visão, valores, estratégias e } \\
\text { Metas aos seus gestores }\end{array}$} & Quant. & 11 & 0 & \multirow{2}{*}{11} \\
\hline & $\%$ & $100 \%$ & $0 \%$ & \\
\hline \multirow{2}{*}{$\begin{array}{l}\text { Apresenta claramente sua visão, valores, estratégias e } \\
\text { Metas aos seus diretores }\end{array}$} & Quant. & 10 & 1 & \multirow{2}{*}{11} \\
\hline & $\%$ & $91 \%$ & $9 \%$ & \\
\hline \multirow{2}{*}{$\begin{array}{l}\text { Apresenta claramente sua visão, valores, estratégias e } \\
\text { Metas aos seus funcionários }\end{array}$} & Quant. & 10 & 1 & \multirow{2}{*}{11} \\
\hline & $\%$ & $91 \%$ & $9 \%$ & \\
\hline \multirow{2}{*}{ Utiliza relatórios financeiros e não-financeiros } & Quant. & 10 & 1 & \multirow{2}{*}{11} \\
\hline & $\%$ & $91 \%$ & $9 \%$ & \\
\hline \multirow{2}{*}{ Utiliza sistema de medição de desempenho } & Quant. & 7 & 4 & \multirow{2}{*}{11} \\
\hline & $\%$ & $64 \%$ & $36 \%$ & \\
\hline \multirow{2}{*}{ Realiza Benchmarking } & Quant. & 3 & 8 & \multirow{2}{*}{11} \\
\hline & $\%$ & $27 \%$ & $73 \%$ & \\
\hline \multirow{2}{*}{$\begin{array}{l}\text { Os indicadores de desempenho atualmente auxiliam na } \\
\text { tomada de decisão da empresa? }\end{array}$} & Quant. & 9 & 2 & \multirow{2}{*}{11} \\
\hline & $\%$ & $82 \%$ & $18 \%$ & \\
\hline \multirow{2}{*}{ 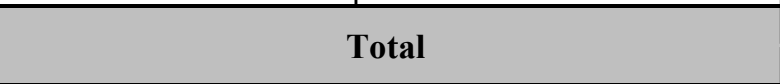 } & Quant. & 92 & 18 & 110 \\
\hline & $\%$ & $83,64 \%$ & $16,36 \%$ & $100 \%$ \\
\hline
\end{tabular}

Fonte: Dados da Pesquisa, 2016 
Verificou-se, na tabela 3, que $100 \%$ das empresas elaboram orçamento, realizam comparações entre o planejado e executado e apresentam claramente sua visão, valores, estratégias e metas aos seus gestores. Já os aspectos Possuir missão definida; apresentar claramente sua visão, valores, estratégias e metas aos seus diretores e funcionários; e utilizar relatórios financeiros e não-financeiros são práticas adotadas por $91 \%$ da empresas. Os indicadores de desempenho atualmente auxiliam na tomada de decisão em $82 \%$ das empresas estudadas. Porém, do total dos respondentes, apenas $64 \%$ disseram utilizar sistema de medição de desempenho e $73 \%$ afirmam não realizar benchmarking. Dessa forma, infere-se que $83,64 \%$ das atividades relacionadas com a medição de desempenho são realizadas pelos gestores.

Em relação à importância de possuir um sistema de desempenho, constatou-se, conforme tabela 4, que os itens considerados de maior relevância foram o fato de poder controlar as atividades operacionais da empresa e verificar se a missão da empresa está sendo atingida, ambos com $73 \%$ de representatividade; seguido, com 55\%, da identificação de problemas que necessitem a intervenção dos gestores; $45 \%$ para controlar planejamento e criar, implantar e conduzir estratégias competitivas; e, por último, com $36 \%$ dos respondentes, o item alimentar os sistemas de incentivo dos funcionários.

Tabela 4: Importância identificada para possuir um sistema de desempenho

\begin{tabular}{|c|c|c|c|c|c|c|}
\hline Alternativas & & $\mathbf{I}$ & II & III & IV & Total \\
\hline \multirow{2}{*}{ Controlar as atividades operacionais da empresa } & Quant. & 0 & 1 & 2 & 8 & \multirow{2}{*}{11} \\
\hline & $\%$ & $0 \%$ & $9 \%$ & $18 \%$ & $73 \%$ & \\
\hline \multirow{2}{*}{ Alimentar os sistemas de incentivo dos funcionários } & Quant. & 0 & 5 & 2 & 4 & \multirow{2}{*}{11} \\
\hline & $\%$ & $0 \%$ & $45 \%$ & $18 \%$ & $36 \%$ & \\
\hline \multirow{2}{*}{ Controlar o planejamento } & Quant. & 0 & 1 & 5 & 5 & \multirow{2}{*}{11} \\
\hline & $\%$ & $0 \%$ & $9 \%$ & $45 \%$ & $45 \%$ & \\
\hline \multirow{2}{*}{ Criar, implantar e conduzir estratégias competitivas. } & Quant. & 0 & 1 & 5 & 5 & \multirow{2}{*}{11} \\
\hline & $\%$ & $0 \%$ & $9 \%$ & $45 \%$ & $45 \%$ & \\
\hline \multirow{2}{*}{$\begin{array}{l}\text { Identificar problemas que necessitem intervenção dos } \\
\text { gestores }\end{array}$} & Quant. & 0 & 2 & 3 & 6 & \multirow{2}{*}{11} \\
\hline & $\%$ & $0 \%$ & $18 \%$ & $27 \%$ & $55 \%$ & \\
\hline \multirow{2}{*}{ Verificar se a missão da empresa está sendo atingida } & Quant. & 1 & 0 & 2 & 8 & \multirow{2}{*}{11} \\
\hline & $\%$ & $9 \%$ & $0 \%$ & $18 \%$ & $73 \%$ & \\
\hline \multirow{2}{*}{ Total } & Quant. & 1 & 10 & 19 & 36 & 66 \\
\hline & $\%$ & $2 \%$ & $15 \%$ & $29 \%$ & $55 \%$ & $100 \%$ \\
\hline
\end{tabular}

Para I- Não considera importante; II- Pouco importante; III- Importância razoável; IV- Muito importante.

Fonte: Dados da Pesquisa, 2016

Adentrando nas barreiras apontadas pelos gestores (tabela 5), no que se refere à implantação de um sistema de indicadores de desempenho, destacam-se, como as mais importantes, o custobenefício, com 64\%; seguida das quantidades de indicadores, falta de motivação por parte dos funcionários e dificuldade de elaborarem os objetivos e estratégias, com $27 \%$; e as demais, como o tempo para coletar os dados, dificuldade para analisar os dados e falta de conhecimento sobre o que medir e como medir, contaram com $18 \%$ das opiniões. 
Tabela 5: Barreiras para implantar um sistema de indicadores de desempenho

\begin{tabular}{|c|c|c|c|c|c|c|}
\hline Alternativas & & $\mathbf{I}$ & II & III & IV & Total \\
\hline \multirow{2}{*}{ Tempo para coletar os dados } & Quant. & 3 & 3 & 3 & 2 & \multirow{2}{*}{11} \\
\hline & $\%$ & $27 \%$ & $27 \%$ & $27 \%$ & $18 \%$ & \\
\hline \multirow{2}{*}{ Dificuldades para analisar os dados } & Quant. & 2 & 2 & 5 & 2 & \multirow{2}{*}{11} \\
\hline & $\%$ & $18 \%$ & $18 \%$ & $45 \%$ & $18 \%$ & \\
\hline \multirow{2}{*}{ Quantidades de indicadores } & Quant. & 0 & 4 & 4 & 3 & \multirow{2}{*}{11} \\
\hline & $\%$ & $0 \%$ & $36 \%$ & $36 \%$ & $27 \%$ & \\
\hline \multirow{2}{*}{ Falta de motivação por parte dos funcionários } & Quant. & 1 & 2 & 5 & 3 & \multirow{2}{*}{11} \\
\hline & $\%$ & $9 \%$ & $18 \%$ & $45 \%$ & $27 \%$ & \\
\hline \multirow{2}{*}{ Falta de interesse pela direção } & Quant. & 3 & 1 & 4 & 3 & \multirow{2}{*}{11} \\
\hline & $\%$ & $27 \%$ & $9 \%$ & $36 \%$ & $27 \%$ & \\
\hline \multirow{2}{*}{ Custo-benefício } & Quant. & 2 & 1 & 1 & 7 & \multirow{2}{*}{11} \\
\hline & $\%$ & $18 \%$ & $9 \%$ & $9 \%$ & $64 \%$ & \\
\hline \multirow{2}{*}{ Dificuldade de elaborarem os objetivos e estratégias } & Quant. & 3 & 1 & 4 & 3 & \multirow{2}{*}{11} \\
\hline & $\%$ & $27 \%$ & $9 \%$ & $36 \%$ & $27 \%$ & \\
\hline \multirow{2}{*}{ Falta de conhecimento sobre o que medir e como medir } & Quant. & 3 & 1 & 5 & 2 & \multirow{2}{*}{11} \\
\hline & $\%$ & $27 \%$ & $9 \%$ & $45 \%$ & $18 \%$ & \\
\hline \multirow{2}{*}{ Total } & Quant. & 17 & 15 & 31 & 25 & 88 \\
\hline & $\%$ & $19 \%$ & $17 \%$ & $35 \%$ & $28 \%$ & $100 \%$ \\
\hline
\end{tabular}

Para I- Não considera importante; II- Pouco importante; III- Importância razoável; IV- Muito importante.

Fonte: Dados da Pesquisa, 2016

Já os fatores tempo para coletar os dados, falta de interesse da direção, dificuldade de elaboração dos objetivos e estratégias, e falta de conhecimento sobre o que medir e como medir, os indicadores representavam $27 \%$ das justificativas não consideradas importantes para impedir a implantação de um sistema de indicadores de desempenho. Os quesitos contemplados, nessa parte do questionário, tiveram uma distribuição dos totais, em nível de importância, bem moderada, sobressaindo, com 35\%, a importância razoável.

Em seguida, apresentado no quadro 1, estão as frequências de utilização dos indicadores, sob a perspectiva de processo interno de negócios, contemplada pelo Balanced Scorecard, e sua distribuição percentual. Observou-se que o indicador com maior grau de utilização (utiliza sempre), evidenciado na pesquisa, foi a produtividade global da obra, com 55\%, programação concluída e velocidade de vendas, com $36 \%$. Além desses indicadores, o retrabalho, a velocidade de vendas e tempo de lançamento de novos produtos são utilizados com frequência, segundo os $55 \%$ dos respondentes. Já o indicador de faturamento ou lucro por empregado, acidentes de trabalho com afastamento, e faturamento aplicado na aquisição de equipamentos foram os mais citados como não utilizados pelos gestores, com 36\%. Contudo, a vertente "utilização frequentemente" foi superior aos demais níveis de utilização, com 39\%. 
Quadro 1: Utilização dos indicadores de desempenho na perspectiva do BSC

\begin{tabular}{|c|c|c|c|c|c|c|c|}
\hline & Alternativas & & $\begin{array}{c}\text { Não } \\
\text { Utiliza }\end{array}$ & $\begin{array}{l}\text { Utiliza } \\
\text { Rara- } \\
\text { mente }\end{array}$ & $\begin{array}{c}\text { Utiliza } \\
\text { Frequen- } \\
\text { temente }\end{array}$ & $\begin{array}{l}\text { Utiliza } \\
\text { sempre }\end{array}$ & Total \\
\hline \multirow{22}{*}{ 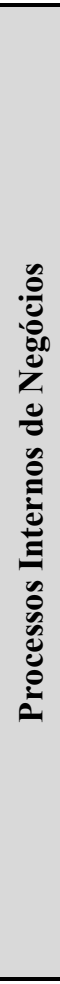 } & \multirow{2}{*}{ Percentual de programação concluída } & Quant. & 1 & 3 & 3 & 4 & \multirow{2}{*}{11} \\
\hline & & $\%$ & $9 \%$ & $27 \%$ & $27 \%$ & $36 \%$ & \\
\hline & \multirow{2}{*}{ Modificação de projeto } & Quant. & 2 & 2 & 4 & 3 & \multirow{2}{*}{11} \\
\hline & & $\%$ & $18 \%$ & $18 \%$ & $36 \%$ & $27 \%$ & \\
\hline & \multirow{2}{*}{ Avaliação de fornecedores } & Quant. & 3 & 2 & 4 & 2 & \multirow{2}{*}{11} \\
\hline & & $\%$ & $27 \%$ & $18 \%$ & $36 \%$ & $18 \%$ & \\
\hline & \multirow{2}{*}{ Produtividade global da obra } & Quant. & 2 & 2 & 1 & 6 & \multirow{2}{*}{11} \\
\hline & & $\%$ & $18 \%$ & $18 \%$ & $9 \%$ & $55 \%$ & \\
\hline & \multirow{2}{*}{ Retrabalho } & Quant. & 3 & 1 & 6 & 1 & \multirow{2}{*}{11} \\
\hline & & $\%$ & $27 \%$ & $9 \%$ & $55 \%$ & $9 \%$ & \\
\hline & \multirow{2}{*}{ Velocidade de Vendas } & Quant. & 1 & 0 & 6 & 4 & \multirow{2}{*}{11} \\
\hline & & $\%$ & $9 \%$ & $0 \%$ & $55 \%$ & $36 \%$ & \\
\hline & \multirow{2}{*}{ Padronização dos processos } & Quant. & 2 & 1 & 5 & 3 & \multirow{2}{*}{11} \\
\hline & & $\%$ & $18 \%$ & $9 \%$ & $45 \%$ & $27 \%$ & \\
\hline & \multirow{2}{*}{ Faturamento ou Lucro por Empregado } & Quant. & 4 & 1 & 5 & 1 & \multirow{2}{*}{11} \\
\hline & & $\%$ & $36 \%$ & $9 \%$ & $45 \%$ & $9 \%$ & \\
\hline & \multirow{2}{*}{$\begin{array}{l}\text { Tempo de Lançamento de novo } \\
\text { empreendimento }\end{array}$} & Quant. & 2 & 0 & 6 & 3 & \multirow{2}{*}{11} \\
\hline & & $\%$ & $18 \%$ & $0 \%$ & $55 \%$ & $27 \%$ & \\
\hline & \multirow{2}{*}{ Acidentes de Trabalho com Afastamento } & Quant. & 4 & 1 & 4 & 2 & \multirow{2}{*}{11} \\
\hline & & $\%$ & $36 \%$ & $9 \%$ & $36 \%$ & $18 \%$ & \\
\hline & \multirow{2}{*}{$\begin{array}{l}\text { Faturamento Aplicado na Aquisição de } \\
\text { Equipamentos/tecnologia }\end{array}$} & Quant. & 4 & 1 & 3 & 3 & \multirow{2}{*}{11} \\
\hline & & $\%$ & $36 \%$ & $9 \%$ & $27 \%$ & $27 \%$ & \\
\hline & \multirow{2}{*}{ Total } & Quant. & 28 & 14 & 47 & 32 & 121 \\
\hline & & $\%$ & $23 \%$ & $12 \%$ & $39 \%$ & $26 \%$ & $100 \%$ \\
\hline
\end{tabular}

Fonte: Dados da Pesquisa, 2016

Considerando a perspectiva financeira (quadro 2), o indicador com maior grau de utilização (utiliza sempre), exposto na pesquisa, foi o custo total da obra, com $64 \%$, e fluxo de caixa, com uma representatividade de 55\%; os demais se mantiveram na média de 18 e $27 \%$.

Quadro 2: Utilização dos indicadores de desempenho na perspectiva do BSC

\begin{tabular}{|c|c|c|c|c|c|c|c|}
\hline & Alternativas & & $\begin{array}{l}\text { Não } \\
\text { Utiliza }\end{array}$ & $\begin{array}{l}\text { Utiliza } \\
\text { Rara- } \\
\text { mente }\end{array}$ & $\begin{array}{c}\text { Utiliza } \\
\text { Frequen- } \\
\text { temente }\end{array}$ & $\begin{array}{l}\text { Utiliza } \\
\text { sempre }\end{array}$ & Total \\
\hline \multirow{18}{*}{ 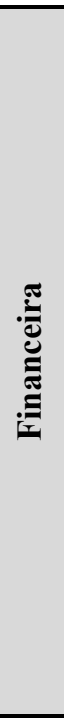 } & \multirow{2}{*}{ EBITDA } & Quant. & 2 & 3 & 4 & 2 & \multirow{2}{*}{11} \\
\hline & & $\%$ & $18 \%$ & $27 \%$ & $36 \%$ & $18 \%$ & \\
\hline & \multirow{2}{*}{ Fluxo de Caixa } & Quant. & 0 & 3 & 2 & 6 & \multirow{2}{*}{11} \\
\hline & & $\%$ & $0 \%$ & $27 \%$ & $18 \%$ & $55 \%$ & \\
\hline & \multirow{2}{*}{$\begin{array}{l}\text { Composição de Endividamento (Passivo } \\
\text { Circulante/Passivo Total) }\end{array}$} & Quant. & 2 & 2 & 4 & 3 & \multirow{2}{*}{11} \\
\hline & & $\%$ & $18 \%$ & $18 \%$ & $36 \%$ & $27 \%$ & \\
\hline & \multirow{2}{*}{$\begin{array}{l}\text { Retorno sobre Investimento- ROI (Lucro } \\
\text { Operacional/Investimento Médio) }\end{array}$} & Quant. & 2 & 1 & 6 & 2 & \multirow{2}{*}{11} \\
\hline & & $\%$ & $18 \%$ & $9 \%$ & $55 \%$ & $18 \%$ & \\
\hline & \multirow{2}{*}{$\begin{array}{l}\text { Retorno Sobre o Ativo- ROA (Lucro } \\
\text { Operacional/Ativo Total Médio) }\end{array}$} & Quant. & 2 & 1 & 6 & 2 & \multirow{2}{*}{11} \\
\hline & & $\%$ & $18 \%$ & $9 \%$ & $55 \%$ & $18 \%$ & \\
\hline & \multirow{2}{*}{$\begin{array}{l}\text { Retorno Sobre o Patrimôno Líquido - ROE } \\
\text { (Lucro Líquido/PL Médio) }\end{array}$} & Quant. & 2 & 1 & 6 & 2 & \multirow{2}{*}{11} \\
\hline & & $\%$ & $18 \%$ & $9 \%$ & $55 \%$ & $18 \%$ & \\
\hline & \multirow{2}{*}{ Margem de Lucro (Lucro Líquido/ Vendas) } & Quant. & 1 & 0 & 7 & 3 & \multirow{2}{*}{11} \\
\hline & & $\%$ & $9 \%$ & $0 \%$ & $64 \%$ & $27 \%$ & \\
\hline & \multirow{2}{*}{$\begin{array}{l}\text { Lucratividade [(Lucro Líquido/ Receita Bruta) } \\
\text { x } 100\end{array}$} & Quant. & 1 & 0 & 7 & 3 & \multirow{2}{*}{11} \\
\hline & & $\%$ & $9 \%$ & $0 \%$ & $64 \%$ & $27 \%$ & \\
\hline & \multirow{2}{*}{ Custo Total da Obra } & Quant. & 1 & 0 & 3 & 7 & \multirow{2}{*}{11} \\
\hline & & $\%$ & $9 \%$ & $0 \%$ & $27 \%$ & $64 \%$ & \\
\hline & \multirow{2}{*}{ Total } & Quant. & 13 & 11 & 45 & 30 & 99 \\
\hline & & $\%$ & $13 \%$ & $11 \%$ & $45 \%$ & $30 \%$ & $100 \%$ \\
\hline
\end{tabular}

Fonte: Dados da Pesquisa, 2016 
Os indicadores lucratividade e margem de lucro mostraram que são utilizados frequentemente por $64 \%$ dos respondentes, e os três indicadores de rentabilidade (retorno) com $55 \%$. No máximo, $18 \%$ dos gestores deixam de utilizar alguns dos indicadores levantados no estudo. Vale destacar que, assim como ocorreu com a perspectiva anterior, a classificação "utilização frequentemente" foi superior aos demais níveis de utilização, com $45 \%$.

Quadro 3: Utilização dos indicadores de desempenho na perspectiva do BSC

\begin{tabular}{|c|c|c|c|c|c|c|c|}
\hline & Alternativas & & $\begin{array}{c}\text { Não } \\
\text { Utiliza }\end{array}$ & $\begin{array}{c}\text { Utiliza Rara- } \\
\text { mente }\end{array}$ & $\begin{array}{c}\text { Utiliza } \\
\text { Frequentemente }\end{array}$ & $\begin{array}{l}\text { Utiliza } \\
\text { sempre }\end{array}$ & Total \\
\hline \multirow{8}{*}{ } & \multirow{2}{*}{ Satisfação do Cliente } & Quant. & 3 & 1 & 4 & 3 & \multirow{2}{*}{11} \\
\hline & & $\%$ & $27 \%$ & $9 \%$ & $36 \%$ & $27 \%$ & \\
\hline & \multirow{2}{*}{ Número de Clientes } & Quant. & 1 & 1 & 5 & 4 & \multirow{2}{*}{11} \\
\hline & & $\%$ & $9 \%$ & $9 \%$ & $45 \%$ & $36 \%$ & \\
\hline & \multirow{2}{*}{ Cota de Mercado } & Quant. & 3 & 2 & 5 & 1 & \multirow{2}{*}{11} \\
\hline & & $\%$ & $27 \%$ & $18 \%$ & $45 \%$ & $9 \%$ & \\
\hline & \multirow{2}{*}{ Pontualidade na Entrega } & Quant. & 1 & 0 & 4 & 6 & \multirow{2}{*}{11} \\
\hline & & $\%$ & $9 \%$ & $0 \%$ & $36 \%$ & $55 \%$ & \\
\hline & \multirow{2}{*}{ Total } & Quant. & 8 & 4 & 18 & 14 & 44 \\
\hline & & $\%$ & $18 \%$ & $9 \%$ & $41 \%$ & $32 \%$ & $100 \%$ \\
\hline
\end{tabular}

Fonte: Dados da Pesquisa, 2016

Quanto à perspectiva do cliente, os resultados demonstraram uma concentração maior da classificação "utiliza frequentemente" (41\%) e "utiliza sempre" (32\%), como pode ser verificado no quadro 3. Na primeira situação, os indicadores de número de clientes $(45 \%)$ e cota de mercado (45\%) foram os mais pontuados, enquanto na segunda classificação foram enquadrados os itens pontualidade na entrega (55\%) e número de clientes (36\%). Percebe-se, ainda, que $27 \%$ dos gestores não utilizam o indicar satisfação do cliente e cota de mercado.

Em relação à perspectiva de aprendizagem e crescimento, observou-se um comportamento adverso das demais perspectivas apresentadas, pois houve uma maior incidência de "não utiliza" indicadores, conforme apresentado no quadro 4.

\begin{tabular}{|c|c|c|c|c|c|c|c|}
\hline & Alternativas & & $\begin{array}{c}\text { Não } \\
\text { Utiliza }\end{array}$ & $\begin{array}{l}\text { Utiliza } \\
\text { Rara- } \\
\text { mente }\end{array}$ & $\begin{array}{c}\text { Utiliza } \\
\text { Frequen- } \\
\text { temente }\end{array}$ & $\begin{array}{c}\text { Utiliza } \\
\text { sempre }\end{array}$ & Total \\
\hline \multirow{8}{*}{ 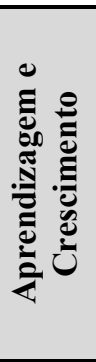 } & \multirow{2}{*}{ Número de Treinamento } & Quant. & 5 & 2 & 3 & 1 & \multirow{2}{*}{11} \\
\hline & & $\%$ & $45 \%$ & $18 \%$ & $27 \%$ & $9 \%$ & \\
\hline & \multirow{2}{*}{ Motivação após Treinamento } & Quant. & 4 & 4 & 3 & 0 & \multirow{2}{*}{11} \\
\hline & & $\%$ & $36 \%$ & $36 \%$ & $27 \%$ & $0 \%$ & \\
\hline & \multirow{2}{*}{ Perspectivas dos Colaboradores } & Quant. & 5 & 3 & 3 & 0 & \multirow{2}{*}{11} \\
\hline & & $\%$ & $45 \%$ & $27 \%$ & $27 \%$ & $0 \%$ & \\
\hline & \multirow{2}{*}{ Satisfação dos Funcionários } & Quant. & 5 & 2 & 4 & 0 & \multirow{2}{*}{11} \\
\hline & & $\%$ & $45 \%$ & $18 \%$ & $36 \%$ & $0 \%$ & \\
\hline & \multirow{2}{*}{ Total } & Quant. & 19 & 11 & 13 & 1 & 44 \\
\hline & & $\%$ & $43 \%$ & $25 \%$ & $30 \%$ & $2 \%$ & $100 \%$ \\
\hline
\end{tabular}

Fonte: Dados da Pesquisa, 2016

No contexto geral, foi possível verificar, sob a ótica do BSC, que os indicadores listados nas perspectivas de processos internos de negócios, financeira e clientes, em termos proporcionais, são "utilizados frequentemente" pelos gestores. Já na perspectiva de aprendizagem e crescimento, 43\% do total dos indicadores não são utilizados pelos gestores. 


\section{CONSIDERAÇÕES FINAIS}

A motivação para aplicação do estudo justifica-se pela importância que o setor de Construção Civil exerce nos aspectos econômicos, sociais, tecnológicos e governamentais do país, e pelo fato do desenvolvimento na construção civil, nas últimas décadas, ter aumentado a competitividade, fazendo com que as empresas repensem suas estratégias, e desenvolvam além da perspectiva financeira. Nesse contexto, a presente pesquisa teve como objetivo analisar, sob a ótica do BSC, os indicadores de desempenho utilizados pelos gestores das empresas de construção civil do município de João PessoaPB.

Os resultados obtidos sob a perspectiva de processo interno de negócios, contemplada pelo Balanced Scorecard, e sua distribuição percentual, demonstraram que os indicadores utilizados com maior frequência são a produtividade global da obra, com 55\%, programação concluída e velocidade de vendas, com $36 \%$. Na perspectiva financeira, o indicador com maior grau de utilização, exposto na pesquisa, foi o custo total da obra, com 64\%. Quanto à perspectiva do cliente, os resultados demonstraram que a pontualidade na entrega (55\%) é o principal indicador utilizado para mensurar essa categoria. Em relação à perspectiva de aprendizagem e crescimento, observou-se um comportamento adverso das demais perspectivas apresentadas, visto que $68 \%$ dos gestores dizem não utilizar ou utilizar pouco dos indicadores elencados nessa perspectiva.

Percebe-se, ainda, que os indicadores listados nas perspectivas de processos internos de negócios, financeira e clientes, em termos proporcionais, são "utilizados frequentemente" pelos gestores. Também, sugere-se, com os resultados apresentados, que as empresas precisam reconhecer mais a importância dos direcionadores no desempenho estratégico futuro, ora representado pelos indicadores contemplados na perspectiva de aprendizado e crescimento, pois estes são a base para a melhoria da qualidade, e, por consequência, diferencial na competitividade; entretanto, $43 \%$ dos itens listados, nessa vertente, não são utilizados pelos gestores, mesmo quando estes afirmam, em sua grande maioria, fazer uso de indicadores de desempenho não financeiro.

É relevante apontar, quanto às conclusões deste estudo, que o universo da pesquisa limitou-se apenas 7,09\% das empresas de Construção Civil de João Pessoa, não possibilitando inferências além do universo pesquisado. Partindo desse pressuposto, fica como sugestão para futuros trabalhos, a ampliação da amostra.

\section{REFERÊNCIAS}

ATKINSON, A. A.; WATERHOUSE, J. H.; WELL, R.B.. A stakeholder approach to strategic performance measurement. Sloan Management Review Spring, p. 25-37, 1997.

BOURNE, M.; MILLS, J.; WILCOXS, M.; NEELY, A; PLATTS, K. Designing, implementing and updating performance measurement System. International Journal of Operations \& Production Management, v. 20, n. 7, p. 754-771, 2000.

CALLADO, A. A. C.; JACK, L. Balanced scorecard metrics and specific supply chain roles. International Journal of Productivity \& Performance Measurement, v. 64, n. 2, p. 288-300, 2015.

CHIA, A.; GOH, M.; HUM, S-H. Performance measurement in supply chain entities: balanced scorecard perspective. Benchmarking: An International Journal, v. 16, n. 5, p. 605-620, 2009.

DINIZ, J. L. P.; GODOY, L. P.; STEFANO, N. M.. O uso da ferramenta Balanced scorecardcom ênfase na satisfação dos clientes em uma cooperativa de pequeno porte. Revista Eletrônica de Gestão Organizacional, v. 10, n.3, p. 653-684, 2012.

ECCLES, R. G.. The performance measurement manifesto. Harvard Business Review, JanuaryFebruary, p. 131-137, 1991. 
FILGUEIRAS, A. A; BARROS, L. P. S.; GOMES, J. S. O processo de implantação do Balanced scorecardem uma empresa estatal brasileira: O caso Petrobras. Revista de Gestão, São Paulo, v. 17, n. 1, p. 45-57, jan./mar, 2010.

FOLAN, P.; BROWNE, J. A review of performance measurement: towards performance management. Computers in Industry, v. 56, n. 7, p. 663-680, 2005.

HO, K.; MCKAY, R. Balanced scorecard: two perspectives. The CPA Journal, v. 72, n.3, p. 20-5, 2002.

HOLANDA, F. M. A. Indicadores de desempenho: uma análise nas empresas de construção civil do município de João Pessoa - PB. Dissertação (Mestrado em Ciências Contábeis), Programa Multiinstitucional e Inter-Regional de Pós-Graduação em Ciências Contábeis, João Pessoa, 2007.

KAPLAN, R. S.; NORTON, D. P. A estratégia em ação: Balanced scorecard. 4. ed. Rio de Janeiro: Campus, 1997.

KAPLAN, R. S.; NORTON, D. P. The balanced scorecard- measures that drive performance. Harvard Business Review, V. 70, January/February, 1992.

KAPLAN, R. S.; NORTON, D. P. Transforming the balanced scorecard from performance measurement to strategic management: Part II. Accounting Horizons, v.15, n. 2, p. 147 160, Jun. $2001 b$.

KAPLAN, R. S.; NORTON, D. P. Mapas Estratégicos. Convertendo Ativos Intangíveis em Resultados Tangíveis. 4.ed. Rio de Janeiro: Campus, 2004.

78, n. 5, p. 167-176, sept./oct. 2000a.

Having Trouble With Your Strategy? Then map it. Harvard Business Review, v. . Linking the balanced scorecardto strategy. California Management Review, V. 39,

n. 1, 1996.

KURESKI, R.; RODRIGUES, R. L.; MORETTO, A. C.; SESSO FILHO, U. A.; HARDT, L. P. A. O macrossetor da construção civil na economia brasileira em 2004. Ambiente Construído, v. 8, n. 1, p. 7-19, 2008.

LANTELME, E. M. V.. Proposta de um sistema de indicadores de qualidade e produtividade para a construção civil. 1994. Dissertação (Mestrado em Engenharia) - Núcleo Orientado para a Inovação da Edificação, Programa de Pós-graduação em Engenharia Civil, Universidade Federal do Rio Grande do Sul, Porto Alegre, 1994.

MARTINS, V. G.; GIRÃO, L. F. A. P.; SILVA FILHO, A. C. C.; ARAUJO, A. O. A utilização de modelos de avaliação de desempenho sob a perspectiva do GECON e do Balanced Scorecard: estudo de caso em uma central de cooperativas de Crédito. Revista Evidenciação Contábil \& Finanças, v.1, n.1, p.99-117, 2013.

MOORAJ, S.; OYON, D.; HOSTETTLER, D. The Balanced Scorecard: a necessary good or an unnecessary evil? European Management Journal, V. 17, n. 5, p. 481-491, 1999.

NAVARRO, Gustavo Pedrosa. Proposta de sistema de indicadores de desempenho para gestão de produção em empreendimento de edificações residenciais. 2005. Dissertação de Mestrado da Universidade Federal do Rio Grande do Sul. Porto Alegre. 2005.

NEELY, A. D.; MILlS, J.; PLATTS, K.; GREGORY, M.; RICHARDS, H. Performance measurement system design: should process based approaches be adopted? International Journal of Production Economics, v.46, n.47, p.423-431, 1996.

NEELY, A. D.; RICHARDS, A. H.; MILLS, J. F.; PLATTS, K. W.; BOURNE, M. C. S.. Designing performance measures: a structured approach, International Journal of Operations \& Production Management, vol. 17, n. 11, p. 1131-53, 1997.

ISSN 2595-7287 | RCGC | UFF | Niterói/RJ | V.1 | n.2 | jul-dez. 2018 | p. 36-51 
NEELY, A.. The performance measurement revolution: why now and what next?. International Journal of Operations \& Production Management, v. 19, n. 2, p. 205-28, 1999.

NORREKLIT, H. The balance on the balanced scorecard: a critical analysis of some of its assumptions. Management Accounting Research, v. 11, n. 1, p. 65-88, 2000.

NORREKLIT, H. The balance on the balanced scorecard a critical analysis of some of its assumptions. Management Accounting Research, n. 11, p.65-88, 2008.

OHASHI, E. A. M.; MELHADO, S. B. A importância dos indicadores de desempenho nas empresas construtoras e incorporadoras com certificação ISO 9001:2000. In: ENCONTRO NACIONAL DE TECNOLOGIA DO AMBIENTE CONSTRUÍDO, São Paulo, 2004.

PARANJAPE, B.; ROSSITER, M.; PANTANO, V. Insights from the Balanced scorecard performance measurement systems: successes, failures and future - A review. Measuring Business Excellence, v. 10, n. 3, p. 4-14, 2006.

PECH, L. M.; RAMOS, F. M.; FAVRETTO, J.; ARTIFON, R. L.. Utilização do balanced scorecard em ambientes cooperativistas: um estudo de caso. REUNA, v.20, n.3, p.05-26, 2015.

PINTO, A. K. GESTÃO ESTRATÉGICA E INDICADORES DE DESEMPENHO. São Paulo, Qualytymark, 2002.

PRIETO, Vanderli Correia; PEREIRA, Fábio Luis Alves; CARVALHO, Marly Monteiro de; LAURINDO, Fernando José Barbin. Fatores Críticos na implementação do Balanced Scorecard. Revista Gestão \& Produção, v.13, n.1, p.81-92, jan./abr., 2006.

PUNNIYAMMOORTHY, M.; MURALI, R. Balanced score for the balanced scorecard; a benchmarking tool. Benchmarking: An International Journal, v. 15, n. 4, p. 420-443, 2008.

ROCHA, J. M.; CASARTElLI, A. O.. Análise do processo de implantação do Balanced scorecard(BSC) em uma instituição de ensino superior. Revista Gestão Universitária na América Latina-GUAL, v.7, n.3, p. 268-290, 2014.

SAINAGHI, R.; PHILLIPS, P.; CORTI, V. Measuring hotel performance: using a balanced scorecard perspectives' approach. International Journal of Hospitality Management, v. 34, p. 150-159, 2013.

SANTOS, D. F. L.. Modelo de Gestão Financeira Aplicada em Empresa do Setor de Construção Civil. Revista TAC, Rio de Janeiro, v. 5, n. 2, art. 2, p. 119-135, Jul./Dez, 2015.

SINK, D. S.; TUTTLE, T. C. Planejamento e medição para performance. Rio de Janeiro: Quality Mark, 1993.

VAN AKEN, E. M.; LETENS, G.; COLEMAN, G. D.; FARRIS, J.; VAN GOUBERGEN, D.. Assessing maturity and effectiveness of enterprise performance measurement systems. International Journal of Productivity \& Performance Measurement, v. 54, n. 5/6, p. 400-418, 2005.

VAN VEEN-DIRKS, P.; WIJN, M. Strategic control: meshing critical success factors with the balanced scorecard. Long Range Planning, V. 35, n. 4, p. 407-427, 2002.

WOULTERS, M.; SPORTEL, M. The role of existing measures in developing and implementing performance measurement systems. International Journal of Operations \& Production Management, V. 25, n. 11 p. 1062-1082, 2005. 
SOBRE AS AUTORAS

RISOLENE ALVES DE MACENA DE ARAÚJO é mestre em Ciências Contábeis pela UFPB e professora substituta na UFPB.

E-mail: risolenee@hotmail.com

LÍVIA MARIA DA SILVA SANTOS é mestre em Ciências Contábeis pela UFPB e Professora Assistente no Centro Universitário de João Pessoa.

E-mail: livia.marias@hotmail.com 